\title{
An Enhanced Approach for Solving Class Imbalance Problem in Automatic Image Annotation
}

\author{
T.Sumadhi \\ Research Scholar, Department of Software Systems, Karpagam University \\ Coimbatore, Tamilnadu, India \\ E-mail: t_sumathi@yahoo.co.in \\ M.Hemalatha \\ Professor\& Head, Department of Software Systems, Karpagam University \\ Coimbatore, Tamilnadu, India \\ E-mail: hema.bioinf@gmail.com
}

\begin{abstract}
Classifying an object captured in an image is useful for understanding the contents of the image and annotating it exactly with corresponding tags automatically is the problem faced recently. As the real world data set is highly imbalanced it degrades the performance of automatic image annotation and object detection. To prevail over this drawback we have proposed a new system for pattern matching and annotation which is based on the fusion of principles obtained from Fractal Transform and gentle AdaBoost algorithm. This paper, also tries to overcome deterioration in the performance occurring through imbalance dataset, different orientation, scaling in image annotation by choosing an over sampling method for learning the classifier. The proposed IFSMOTE classifier is initially trained up by setting a threshold value which helps to identify the objects correctly and an over-sampling technique based on fractal is used to classify the imbalanced dataset. Experimental results on the Flicker image dataset have shown superior performance results in terms of precision, recall and Fmeasure. This paper also presents the comparative results of our proposed system with other traditional image annotation algorithm like SVM, SMOTE and FSMOTE.
\end{abstract}

Index Terms - Automatic image annotation, Gentle Ada-Boost, Improvised FSMOTE, Synthetic minority over sampling technique, JEC, SVM

\section{INTRODUCTION}

Given an input image, the aim of automatic image annotation is to assign a few relevant keywords to the image that reflects its visual content. Utilizing this image content to assign a richer and more relevant set of keywords allow us to develop the fast indexing and retrieval architecture of these search engines for improved image search. The image database retrieval problem involves finding in the database, instances of image content that is similar to the content of interest, specified by the user. The required content can be specified by example, or it can be defined abstractly in terms of concepts. In the former case we refer to the retrieval problem as content based retrieval, and in the latter case as concept-detection. A machine learning algorithm is then used to construct a model of the concept class that can successfully discriminate concept samples from negative (anti-concept) instances. Mathematically, the concept-detection problem can be formulated as either one class or conventional two-class pattern recognition problem. In the former case we build a generative model of the concept class and the conceptdetection then involves testing the hypothesis that an unknown image is consistent with the model. The alternative is to view the concept retrieval as a two-class problem where the second class is represented by negative samples, i.e. images that do not contain the specified concept. The problem can be solved using generative or discriminative models learnt using the training data.

The most common problem faced by inductive learning algorithms on fields for which some classes are represented by a large number of instances while others are represented by only a few instances this is called Imbalanced data sets (IDS) problem or class imbalance problem[7,10,15]. This normally meets two-class problems, which mean one class has much more instances than the other. Occasionally, we might also have multi-class cases, in which there are not sufficient instances for more than one class. It may cause more trouble when deciding classification boundary. Recent machine learning does not provide satisfactory classification performance and so class imbalance learning has received considerable attention nowadays. Traditional classification and categorization algorithms are overwhelmed by majority examples while minority examples contribute very little. A number of enhanced algorithms have been proposed in the review of literature, in which considerations have been made at the data level and algorithm level. 
Many different forms of re-sampling techniques have been proposed at the data level, including over-sampling, under-sampling, and combinations of this both. The results obtained have shown to achieve good performance on minority examples of two-class data sets. The re-sampling techniques have a tendency to degrade when applied to imbalanced data sets with multiple classes. Hence, multi-class classification in imbalanced data sets remains an important topic of research. It has been experimentally observed that class imbalance may create significant deterioration in the performance achieved by existing learning and classification systems. This situation is often found in real-world data describing an infrequent but important case. Recently, the class imbalance problem has received considerable attention in areas such as Machine Learning and Pattern Recognition.

The proposed algorithm accomplishes the following tasks which degrade the performance of automatic image annotation approaches.

- Resampling methods based on fractal theory has been proposed for balancing the data set.

- Modification to the existing learning algorithms has been made to detect the object more accurately.

- The proposed classifier cum learner performance is measured in imbalanced domains.

- The comparative analysis showing the performance of our proposed algorithm with other state-of-art methods is given to show the effectiveness of our algorithm.

The paper is organized as follows. Section 2 provides literature review of several class imbalance methods followed by proposed improvised fractal smote algorithm (IFSMOTE) in section 3. Section 4 describes the experimental setup followed by results and discussion in Section 5. The paper is drawn to conclusion in Section 6.

\section{RELATED WORK}

\section{A. Class Imbalance Learning}

A number of solutions to the class-imbalance problem were previously proposed both at the data and algorithmic levels. At the data level, these solutions include many different forms of re-sampling such as random oversampling with replacement, random under-sampling, focused (or directed) over-sampling where no new examples are created, but the choice of samples to replace is focused rather than random, focused (or directed) under-sampling where the choice of examples to eliminate is focused, over-sampling by generating new samples, and combinations of the above techniques [5]. At the algorithmic level, solutions include adjusting the costs of the various classes so as to counter the class imbalance (cost- sensitive learning) adjusting the probabilistic estimate at the tree leaf (when working with decision trees), adjusting the decision threshold, and recognition-based (one-class learning) rather than discrimination-based (two class) learning.

\section{B. Fractal Theory}

Fractal Theory is proposed by Mandelbrot in 1975; it is first used to describe the geometric shapes that look the same on various scales of magnification [1], in that sense every piece is similar to the whole after some translations or rescaling. It is based on fractal dimension, selfsimilarity, statistical self-similarity and power function, and it researches the complex phenomenon that does not the characteristics of the scale, very irregular and highly fragmented. The core of fractal theory is scale-invariant, or called self-similarity, which means the nature of the object such as shape, structure, information, time remains unchanged in any scales. The self-similarity obey the following power-low: the fractal measure of interest $\boldsymbol{M}(\sigma)$ associates with $\sigma$ as: $\sigma$ as: $M(\sigma) \infty \sigma^{-D}$ where $\infty$ is the meaning of proportionality and $\boldsymbol{D}$ is fractal dimension, $\boldsymbol{D}$ could be any real number[1].

\section{a) Re-sampling}

The basic sampling methods include under-sampling and over-sampling. Under sampling eliminates majorityclass examples while over-sampling increases the number of minority-class examples. Both of these sampling techniques decrease the overall level of class imbalance, thereby making the rare class less rare. These sampling methods have several drawbacks with no doubt. Undersampling discards potentially useful majority-class examples and thus can degrade classifier performance. Therefore, they are normally used on very large data sets in which there are enough redundant data to be removed. Over-sampling, however, can increase the time necessary to build a classifier because it introduces additional training cases. Even worse, because over-sampling often involves making exact copies of examples, it can lead to over fitting $[13,23]$. As an extreme case, final classification rules may be introduced to cover a single, replicated, example. More importantly, over-sampling introduces no new data, so it does not address the fundamental lack of data" issue [2-5].

\section{b) Over-sampling}

\section{1) Random Over-sampling:}

Balance class distribution by replicating minority class examples randomly, but it increases the likelihood of over fitting since it makes exact copies. Make decision regions of the learner more specific and closer to minority class [2, $3,7]$.

\section{2) Smote:}

Generate new synthetic minority examples by interpolating between minority examples that lie together. It makes the decision regions larger towards majority class and less specific. Synthetic examples are introduced along the line segment between each minority class 
example and one of its $\mathrm{k}$ minority class nearest neighbors $[2]$.

\section{3) Fsmote}

The examples SMOTE synthesized are located in the same line; this method makes the synthesized examples obey the spatial distribution to some extent. Fractal Theory is proposed by Mandelbrot in 1982, it is described as a set of "complex" points in a simple space, the structure this points composed has some special properties such as self-similar and symmetry[1-5]. We assume that the data set have the character of self-similar and symmetry too, so we believe that the synthesized examples may not only locate in the same line with another example, but also they can compose similar structure with the original structure [1].

\section{4) Borderline-SMOTE1, borderline-SMOTE2:}

Based on SMOTE algorithm, they generate new minority examples by using the examples only close to decision boundary, and achieve better TP rate and F-value. They considered that examples on the borderline and the ones nearby are more important for classification. First, they find out the borderline minority examples by calculating $\mathrm{k}$ nearest neighbors.

Then SMOTE is applied only to those borderline minority examples to generate new examples. The difference between borderline-SMOTE1 and borderlineSMOTE2 is that the first method generates synthetic examples from original minority examples and their minority nearest neighbors while the other method also generates synthetic examples from their majority nearest neighbors besides minority ones. They experimentally prove that borderline-SMOTE1 behaves good on both recall value and F-value, and borderline-SMOTE2 achieves even better recall value but $\mathrm{F}$-value is decreased because overlap is caused between two classes.[6]

\section{c) Under-sampling}

\section{1) Random Under-sampling:}

Balance class distribution by removing majority class examples randomly. Main disadvantage is that it discards data that may contain useful information. [3]

\section{2) Condensed Nearest Neighbor Rule:}

Condensed Nearest Neighbor Rule (CNN) is used to find a consistent subset of examples by Hart in 1968 . Condensed Nearest Neighbor Rule (CNN) is used to find a consistent subset of examples by Hart in 1968[7, $15,11,12]$.

\section{3) Tomek Link:}

Tomek links was proposed as a data cleaning method in 1976 by Tomek. Given two examples xi and xj belonging to different classes, and $\mathrm{d}(\mathrm{xi} ; \mathrm{xj})$ is the distance between $\mathrm{xi}$ and $\mathrm{xj}$. A (xi; xj) pair is called a Tomek link if there is not an example $x k$, such that $d(x i ; x k)<d(x i ; x j)$ or $d(x j$; $x k)<d(x i ; x j)$. If two examples form a Tomek link, then either one of these examples is noise or both examples are border line. When it is used as an under-sampling method, only examples belonging to the majority class are eliminated, so as to expand borderline towards majority class.

\section{4) One-sided Selection:}

The idea of one-sided selection (OSS) is to remove both redundant and borderline examples from majority class, and keep Isafe" examples for classification. Firstly, redundant examples are removed by using CNN algorithm. Then, borderline examples are followed that participate at Tomek Links.

\section{5) Neighborhood Cleaning Rule:}

Neighborhood cleaning rule uses $\mathrm{kNN}$ to find borderline examples of majority class and remove them. For a two-class problem, for each majority example xi in the training set, its three nearest neighbors are found.

If two or more of them are minority examples, then xi is removed. That means the class of xi contradicts the class given by its nearest neighbors. If $\mathrm{xi}$ belongs to minority class and its nearest neighbors misclassify xi, then the nearest neighbors that belong to majority class are removed. A decontamination methodology is also based on this idea on imbalanced data sets [2].

\section{PROPOSED IFSMOTE CLASSIFIER}

A real-world dataset with two classes is supposed to be imbalanced or skewed when one of the classes (i.e. the minority one) is heavily under-represented in comparison to the other class (i.e. the majority one). This problem is particularly significant in real world applications where it is costly to wrongly classify examples from the minority class, such as information retrieval and filtering tasks, text categorization, detection of fraudulent telephone calls, diagnosis of rare diseases. Normally examples of the minority and majority classes are represented by the absence and presence of rare cases, respectively, by which they are also known as positive and negative examples. As this imbalance dataset which is common in real world data hinders the performance of automatic image annotation we have proposed new solution to solve this problem and thereby increase the performance of annotation of images. Already this technique has been proposed with some limitation in the field of text categorization, but that technique has been altered to overcome those limitations and have been introduced in this field to increase the overall performance of automatic image annotation.

The technique involved in this algorithm performs on two stages in classification phase: first, is the object detection phase for which we make use of gentle Adaboost algorithm as it is best suited for object detection task and also has lower computational complexity. Next, we classify the images based on the mathematical representation which is associated to the fractal interpolation theory for selecting images from the database. This mathematical representation is a set of 
functional parameters resulting from a dedicated fractal interpolation scheme, and has been used as an index by a retrieval algorithm. This proposed new algorithm IFSMOTE is based on the combination of various principles of JEC measure, SVM approach, FSMOTE approach and gentle boost algorithm.

This Process of annotating an image in this method undergoes the following stages:

Step1: Input image is given from the test data set

Step2: The given input image is segmented in to parts based on the features

Step3: Feature are extracted from each segments and from the extracted features we compute JEC and obtain the feature vectors for a particular image

Step4: Based the feature vectors the image is being classified using IFSMOTE algorithm and being annotated.

Step5:Finally we get the annotated image.

Thus the final annotation of an image is obtained at the end of classification.

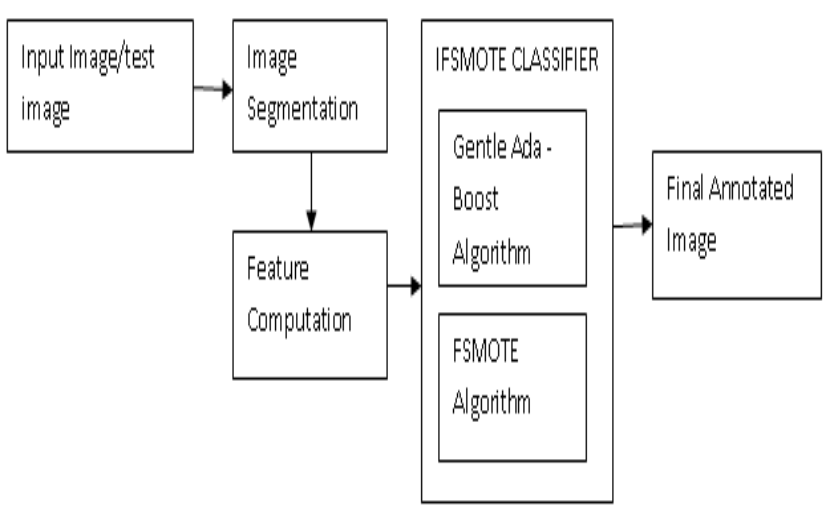

Figure 1: A General Frame work of our proposed system

The IFSMOTE algorithm works as follows:

1. First it creates the necessary data by initializing some parameter values. Mainly it sets up the boosting parameter value also known as the threshold value is used to identify the objects accurately.

2. The three Boosting variants are used for training in each stage. Boosting can be trained on a strong classifier based on a large dataset of weak classifiers by re-weighting the training samples. Weak classifiers are only necessary to be to some extent better than chance. The weak classifier makes use of one feature from the feature pool in combination with a simple binary thresholding decision.

3. At each round of boosting, the Fractal SMOTE classifier is used which best classifies the weighted training samples.
4. By increasing stage number the number of weak classifiers, which are needed to accomplish the preferred false alarm rate at the given hit rate also simultaneously increases.

5. If the preferred hit rate is not obtained then this process is iterated again to step 3 and 4 .

6. Finally, this algorithm evaluates the performance of this classification and annotation performed in terms of precision and recall.

Hence this improved FSMOTE algorithm (IFSMOTE) classifies and annotates the images more accurately, and decreases the error rate in images with different orientation and scale.

Pseudo code for IFSMOTE classifier

\section{Input}

$\mathrm{N}$ : given examples

Wi : the weights; D: JEC distance measure

$\mathrm{x}$ : Query instance to classify

b: Threshold for low-confident prediction

\section{Output}

Final class prediction;

Precision; recall;

Procedure Calls

Gentle Boost

Fsmote classifier

\section{Initializing data}

$\mathrm{x}=\operatorname{rand}(2,1000) ; \mathrm{D}=\operatorname{sqrt}\left(\left((\mathrm{x}(1,:)-.5) \cdot{ }^{\wedge} 2+(\mathrm{x}(2,:)-\right.\right.$ $\left.\left..5) .^{\wedge} 2\right)\right)$

$\mathrm{y}=\mathrm{D}<.2 ; \mathrm{y}=2 * \mathrm{y}-1$

2.. Trainig the classifier using gentle boost algorithm Nrounds $=15$; classifier $=$ gentleBoost $(\mathrm{x}, \mathrm{y}$, Nrounds $)$;

\section{Testing phase of the classifier}

$[\mathrm{x} 1, \mathrm{x} 2]=\operatorname{mesh}$ grid $(0: 0.01: 1,0: 0.01: 1) ;[\mathrm{n}, \mathrm{m}]=$ $\operatorname{size}(\mathrm{x} 1) ; \mathrm{xt}=[\mathrm{x} 1(:) \mathrm{x} 2(:)]^{\prime}$;

\section{Loop on test images}

for $\mathrm{i}=1$ :NtestImages

$\%$ Read image and ground truth

Img = LMimread(data.databaseStruct, testImages(i), HOMEIMAGES);

annotation

data.databaseStruct(testImages(i)).annotation;

5. Normalize the image

[newannotation, newimg, crop, scaling, err, msg] = LMcookimage(annotation, Img, ...

'objectname', objects, 'objectsize', normalizedObjectSize, 'objectlocation', 'original', 'maximagesize', testImageSize);

\section{Running the classifier}

$[\mathrm{Cx}, \mathrm{Fx}]=$ fsmoteclassifier(xt, classifier);

7. Displaying the results

FxShow $=\operatorname{reshape}(\mathrm{Fx},[\mathrm{n} \mathrm{m}])$;

FxShow $=$ FxShow $-\min ($ FxShow $(:))$;

figure

image([0 1], [0 1], 255*FxShow/max(FxShow(:)))

colormap(gray (256)) 
hold on

$\mathrm{j}=\operatorname{find}(\mathrm{y}==-1) ; \operatorname{plot}\left(\mathrm{x}(1, \mathrm{j}), \mathrm{x}(2, \mathrm{j}), \mathrm{rx}^{\prime}\right)$

$\mathrm{j}=$ find $(\mathrm{y}==1) ; \operatorname{plot}\left(\mathrm{x}(1, \mathrm{j}), \mathrm{x}(2, \mathrm{j})\right.$, 'go' $\left.^{\prime}\right)$

title('gentleBoost with stumps')

\section{EXPERIMENTAL SETUP}

Annotation of images in this method undergoes various stages: first, we extract information from the images by using GIST feature descriptor and the extracted features are converted into feature vectors by making use of JEC distance measure and form a pool of a feature vector; next we train IFSMOTE Classifier to create a model for annotation accordingly. Boosting variants are used to train the classifier in each stage. This Boosting can recognize a strong classifier based on a huge set of weak classifiers by re-weighting the training samples. Weak classifiers are only significantly to be slightly superior than chance. The set of weak classifiers make use of one feature vector from pool of feature vectors in combination with a simple binary thresholding decision. Then at each round of boosting, the FSMOTE Classifier is used for the best classification of the weighted training samples. By gradually increasing the stage number the number of weak classifiers, which are desired to achieve the preferred false alarm rate at the given hit rate also simultaneously increases.

\section{A. Data Set Description}

The Flicker dataset which contains 1000 images collected from the larger flicker CD set is used for the experiment. The whole set consists of 10 groups and in each group there are 100 similar images such as beach, aircraft, tiger, car and several indoor images. Each image is annotated with 1-5 words and total of number of keywords in flicker is 415 . It has become the bench mark for image annotation. We divide the dataset into two parts: $90 \%$ images for training and rest $10 \%$ images for testing.

\section{B. Evaluation Measures For Image Annotation.}

To evaluate annotation performance, we query images from the test dataset using 20 frequent keywords from the vocabulary. The image will be retrieved if the automatically established annotation contains the query keyword. We evaluate the result using $\mathrm{P} \%$ and $\mathrm{R} \%$ denotes the mean precision and the mean recall, respectively, over all keywords in percentage points. $\mathrm{N}+$ denotes the number of recalled keywords.

1). Precision is referred as the ratio of the times of correct annotation in relation to all the times of annotation

$$
\text { precision }=\frac{\mid\{\text { relevantimages }\} \cap\{\text { retrievedim ages }\} \mid}{\mid\{\text { retrieve } \operatorname{dim} \text { ages }\} \mid}
$$

2). Recall is referred as the ratio of the times of correct annotation in relation to all the positive samples.

$\operatorname{Re}$ call $=\frac{\mid\{\text { relevantimages }\} \cap\{\text { retrievedim ages }\} \mid}{\mid\{\text { relevantimages }\} \mid}$

3). The weighted harmonic mean of the precision and recall is the F-measure or balanced F-Score is

$$
F=\frac{2 \bullet \operatorname{Pr} \text { ecision } \bullet \text { Re call }}{(\operatorname{Pr} \text { ecision }+\operatorname{Re} \text { call })}
$$

4). The statistical value reflects the coverage of keywords in this proposed method, denoted by "NumWords" N+.

5). The Error measure is E-measure

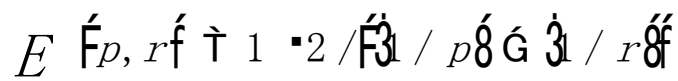

6). Average precision: Precision and recall are singlevalue metrics based on the whole list of images returned by the system. For systems that return a ranked sequence of images, it is desirable to also consider the order in which the returned images are presented. By computing a precision and recall at every position in the ranked sequence of images, we can plot a precision-recall curve, plotting precision $\mathrm{p}(\mathrm{r})$ as a function of recall $\mathrm{r}$. Average precision computes the average value of $p(r)$ over the interval from $r=0$ to $r=1$

$$
A v e P=\sum_{k=1}^{n} P(k) \Delta r(k)
$$

where $\mathrm{k}$ is the rank in the sequence of retrieved images, $\mathrm{n}$ is the number of retrieved images, $\mathrm{P}(\mathrm{k})$ is the precision at cut-off $\mathrm{k}$ in the list, and $\Delta \mathrm{r}(\mathrm{k})$ is the change in recall from items $\mathrm{k}-1$ to $\mathrm{k}$

7). Mean average precision: Mean average precision for a set of queries is the mean of the average precision scores for each query.

$$
M A P=\frac{\sum_{q=1}^{Q} \operatorname{AveP}(q)}{Q}
$$

Where $\mathrm{Q}$ is the number of queries.

\section{V.RESULTS AND DISCUSSION}

In this section we present the results of the proposed method. In order to show the effectiveness of the proposed method a comparative analysis is made with other methods like SVM, HSVM, SMOTE, and FSMOTE. Based on the results obtained as shown in table 1, the proposed method has higher precision, recall and F-measure rates. Figure 2 below shows the objects being identified for the query car side view and monitor screen. Figure 3 below shows the detector output for the 
corresponding input image. The result of precision recall curves for input image is given in Figure 4.

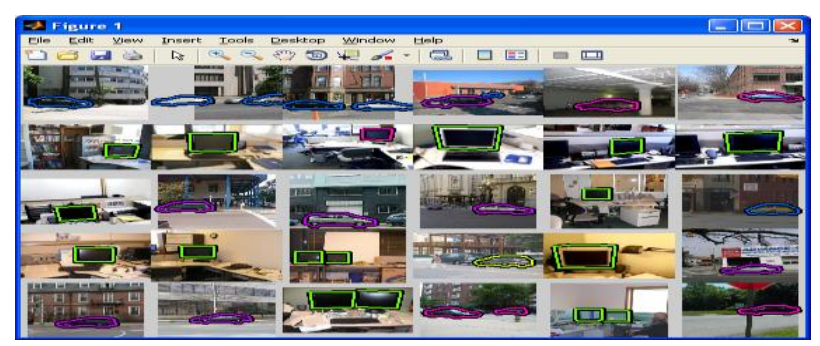

Figure: 2 Retrieval Query based on car side view and Monitor

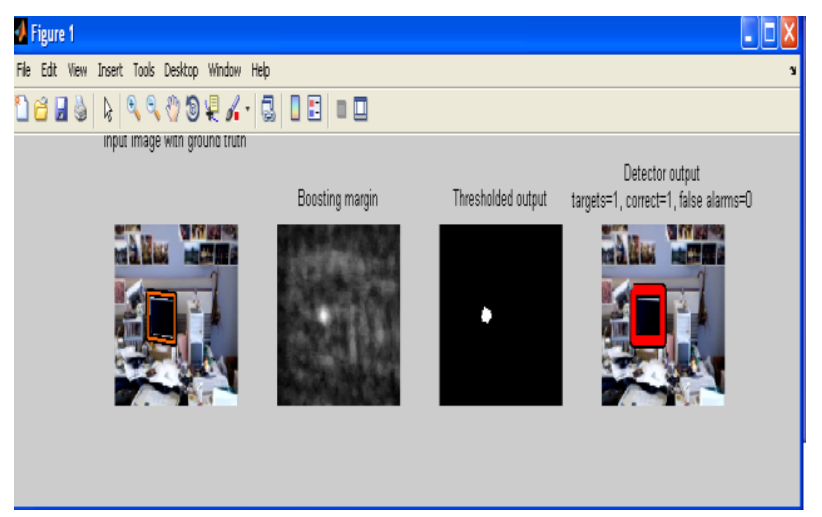

Figure: 3 Detector Output for an input image

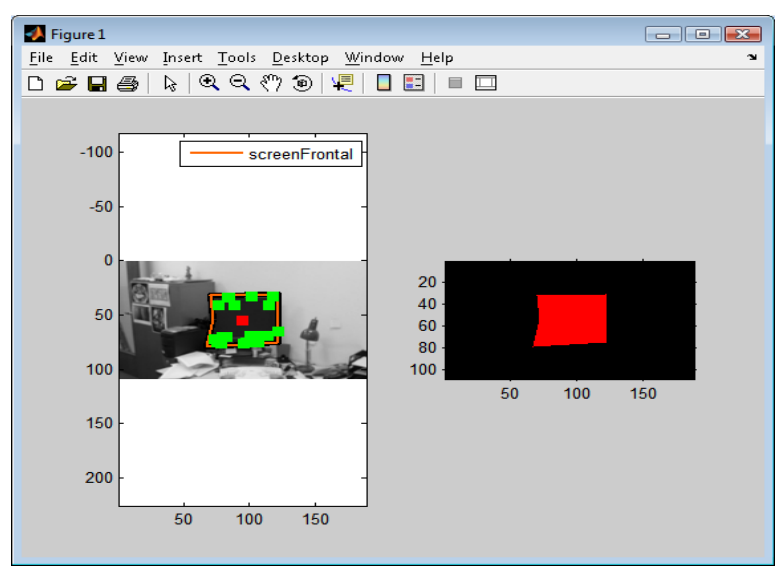

Figure :4 Object detection and annotated for the query of frontal screen

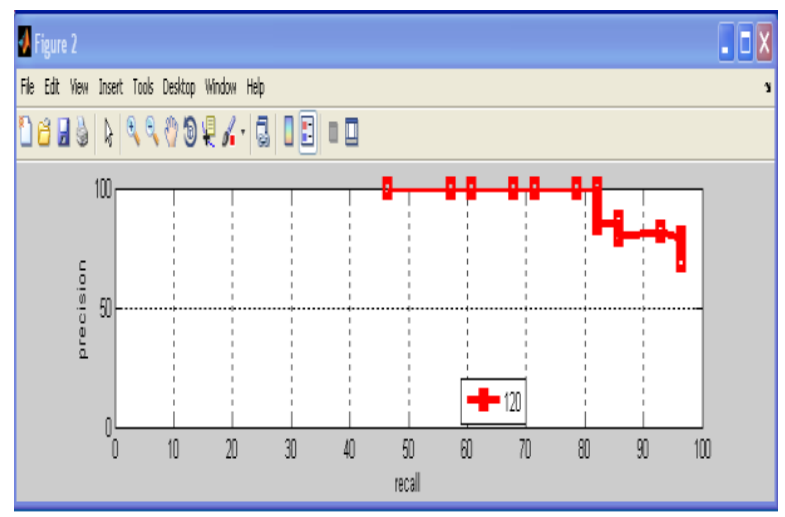

Figure: 5 Precision -Recall Curves for the input image
We have evaluated our proposed method by comparing the results of our method with the previously available methods like SVM, SMOTE, FSMOTE and IFSMOTE classifiers.

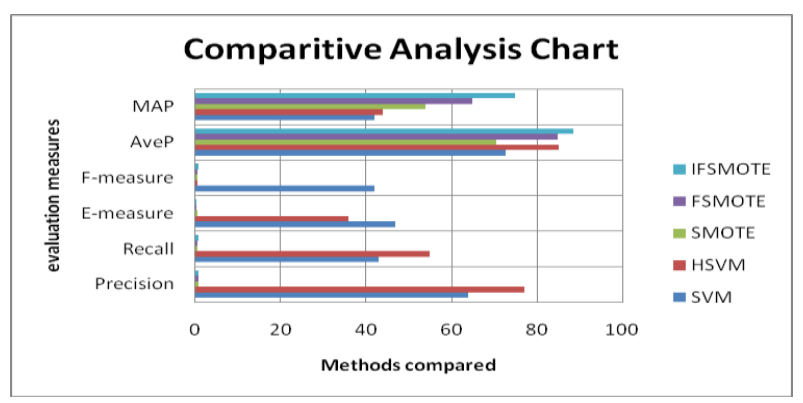

Figure: 6 comparitive analysis chart for the proposed method

Table 1: Comparative results for proposed method

\begin{tabular}{|l|l|l|l|l|l|l|}
\hline Methods & Precision & Recall & $\begin{array}{l}\text { E- } \\
\text { measure }\end{array}$ & $\begin{array}{l}\text { F- } \\
\text { measure }\end{array}$ & AveP & MAP \\
\hline SVM & 0.64 & 0.43 & 0.468 & 0.42 & 72.8 & 42 \\
\hline HSVM & 0.77 & 0.55 & 0.358 & 0.55 & 85.2 & 44 \\
\hline SMOTE & 0.62 & 0.40 & 0.513 & 0.56 & 70.6 & 54 \\
\hline FSMOTE & 0.762 & 0.591 & 0.334 & 0.594 & 85 & 65 \\
\hline IFSMOTE & 0.825 & 0.724 & 0.244 & 0.79 & 88.5 & 75 \\
\hline
\end{tabular}

The results obtained in table 1 clearly show that our proposed IFSMOTE method has higher precision, recall, F-measure and lower e-measure. Hence, we can clearly state that our proposed method outperforms all other state of art methods. We have used both query based retrieval (Fig.2) as well as input image based retrieval. The chart above shows the results of comparison made and we can state our proposed method has higher scores than other methods.

\section{CONCLUSION}

In this paper, a new algorithm has been proposed to solve the class imbalance problem found in annotating an image by detecting the object accurately. Our proposed method also decreases the semantic gap problem by detecting the object for the given query in different orientation and scale. We executed these experiments on flicker data sets, and have made a comparison between the proposed method and traditional classifier algorithm. The result obtained by the proposed IFSMOTE algorithm is better than those obtained by traditional classifier. The main development of this proposed algorithm is that the new synthesized examples agree to the concept of fractal interpolation theory and increases the detection accuracy by using the gentle Ada boost algorithm. The main goal of this work was to design a new annotation algorithm by using the principle based on JEC distance metric, gentle AdaBoost algorithm and FSMOTE. Experiments on these dataset reaffirm the enormous importance of considering multiple sources of evidence to bridge the gap between the pixel representations of images and the semantic meanings. 


\section{REFERENCES}

[1] Dongmei ZHANG, Wei LIU, Xiaosheng GONG, Hui JIN," A Novel Improved SMOTE Resampling Algorithm Based on Fractal" Journal of Computational Information Systems 7: 6 (2011) 2204-2211

[2] Chawla N. V., K. W. Bowyer, L. O. Hall, W. P. Kegelmeyer, 2002. "SMOTE:Synthetic minority oversampling technique," J Artif Intell Res, 16: 321-257.

[3] Tahir M. A., J. Kittler, F. Yan and K. Mikolajczyk" Concept Learning For Image And Video Retrieval: The Inverse Random Under Sampling Approach" European Signal Processing Conference (EUSIPCO 2009) Glasgow, Scotland, August 24-28, 2009.

[4] Shuyun Xie, Zhengyu Bao. Fractal and Multifractal Properties of Geochemical Fields. Earth and Environmental Science. Mathematical Geology. Volume 36,Number 7, 847-864.

[5] Gustavo E. A. P. A. Batista, Ronaldo C. Prati, Maria Carolina Monard, A Study of the Behavior of Several Methods for Balancing Machine Learning Training Data, Sigkdd Explorations, Volume 6, Issue 1 - Page 20.

[6] Chawla, N.V., Lazarevic A., Hall L.O., Bowyer K.W.: SMOTEBoost: Improving Prediction of the Minority Class in Boosting. PKDD 2003, LNAI 2838, pp.107-119, 2003.Springer-Verlag Berlin Heidelberg 2003.

[7] Yang Zhi-ming, Qiao Li-yan, Peng Xi-yuan. Research on Datamining Method for Imbalanced Dataset Based on Improved SMOTE[J]. Acta Electronica Sinica. 2007,35(B12): 22-26.

[8] Chawla, N.V., Data Mining for Imbalanced Datasets: An OverView. In: Maimon, O.,Rokach, L. (eds.) The Data Mining and Knowledge Discovery Handbook, pp. 853-867. Springer, Heidelberg (2005).

[9] L. Jiang and C. Li. An Empirical Study on Attribute Selection Measures in Decision Tree Learning. Journal of Computational Information Systems, 2010, 6(1): 105-112.

[10]Qiong Gu, Zhihua Cai, Li Zhu, Bo Huang. Data Mining on Imbalanced Data Sets. 2008. International Conference on Advanced Computer Theory and Engineering. 1020-1024, 2008.

[11]HeyongWang, Hongkun Fan, Zheng'an Yao. Imbalance Data Set Classification Using SMOTE and Biased-SVM. Computer Science. Vol. 35(5):174-176, 2008.

[12] Sheng TANG and Si-ping CHEN" The Generation Mechanism of Synthetic Minority Class Examples "The 2nd International Symposium \& Summer School on Biomedical and Health Engineering,
China, May 30-31, 978-1-4244-2255-5/08/\$25 2008 IEEE

[13]Benitez A. B. and S.F. Chang. Semantic knowledge construction from annotated image collection. Proceedings of IEEE International Conference on Multimedia, August 2002.

[14] Chang S.F., W. Chen, and H. Sundaram. Semantic visual templates: Linking visual features tosemantics. Proceedings of IEEE International Conference on Image Processing, 1998.

[15]Dietterich T. and G. Bakiri. Solving multiclass learning problems via error-correcting output codes. Journal of Artificial Intelligence Research, 2, 1995.

[16]He X., W.-Y. Ma, O. King, M. Li, and H. Zhang. Learning and inferring a semantic space from user's relevance feedback for image retrieval. Proc. Of ACM Multimedia, :343-347, 2002.

[17]Ho T. K., J. Hull, and S. Srihari. Decision combination in multiple classifier systems. IEEE Transactions on Pattern Analysis and Machine Intelligence, 16(1):66-75, 1994.

[18] J. Li and J. Z. Wang. Automatic linguistic indexing of pictures by a statistical modeling approach. IEEE Transactions on Pattern Analysis and Machine Intelligence, 25(20), 2003.

[19]Lipson P.. Context and configuration based scene classifcation. MIT EECS Department Phd Dissertation, September 1996.

[20]Moreira M. and E. Mayoraz. Improving pairwise coupling classifcation with error correcting classifiers. Proceedings of the Tenth European Conference on Machine Learning, April 1998.

[21]Platt J., N. Cristianini, and J. Shawe-Taylor. Large margin dags for multiclass classification. In Advances in Neural Information Processing Systems 12, pages 547-553. MIT Press, 2000.

[22]Platt J.. Probabilistic outputs for svms and comparisons to regularized likelihood methods. In Advances in Large Margin Classifiers. MIT Press, 1999.

[23]Poddar P. and P. Rao. hierarchical ensemble of neural networks. International Conference on Neural Networks, 1, 1993.

[24] Rodriguez C., J. Muguerza, M. Navarro, A. Zarate, J. Martin, and J. Perez. A two-stage classifier for broken and blurred digits in forms. International Conference on Pattern Recognition, 2:11011105,1998 .

[25] Schapire R. F. and Y. Singer. Improved boosting algorithms using confidence-rated predictions. Proceedings of the 11th Annual Conference on Computational Learning Theory, pages 80-91, July1998. 
[26] Shen H. T., B. C. Ooi, and K. L. Tan. Giving meanings to www images. Proc. of ACM Multimedia: 39-48, 2000.

[27]Smith J. R. and S.F. Chang. Multi-stage classification of images from features and related text. Proc. of the 4th DELOS Workshop, August 1997.

[28] Srihari R., Z. Zhang, and A. Rao. Intelligent indexing and semantic retrieval of multimodal documents. Information Retrieval, 2:245-275, 2000.

[29]Tong S. and E. Chang. Support vector machine active learning for image retrieval. ACM International Conference on Multimedia, October 2001.

[30] Vapnik V.. Estimation of Dependences Based on Empirical Data. Springer Verlag, 1982.

[31] Vapnik V.. Statistical Learning Theory. Wiley, 1998.

[32] Wang J., J. Li, and G. Wiederhold. Simplicity:Semantics-sensitive integrated matching for picture libraries. IEEE Transactions on Pattern Analysis and Machine Intelligence, 23(9):947-963, 2001.

[33] Wang J. Z. and J. Li. Learning-based linguistic indexing of pictures with 2-d mhmms. Proc. Of ACM Multimedia, pages 436-445, December 2002.

[34] Wenyin L., S. Dumais, Y. Sun, H. Zhang, M. Czerwinski, and B. Field. Semi-automatic image annotation. In Proc. of Interact 2001: Conference on Human-Computer Interaction, pages 326-333, Jul 2001.

[35] Wu G. and E. Chang. Adaptive feature-space conformal transformation for learning imbalanced data. International Conference on Machine Learning, August 2003.

\section{AUTHOR PROFILE}

M. Hemalatha completed MCA MPhil., $\mathrm{PhD}$ in Computer Science and currently working as an Assistant Professor and Head, Department of software systems in Karpagam University. Ten years of Experience in teaching and published more than hundred papers in International Journals and also presented seventy papers in various National and international conferences. Area of research is Data mining, Software Engineering, bioinformatics and Neural Network. She is also reviewer in several National and International journals.

T. Sumathi is presently doing $\mathrm{PhD}$ in Karpagam University, Coimbatore, Tamilnadu, India and has completed M.Phil (computer science) in the year 2006 and MCA degree in 1999 and B. Sc(computer science) in 1996. Major research area is Image processing and title for the research is image annotation. At Present, she is working as Lecturer in Karpagam University, Coimbatore and has seven years of Experience in teaching and published more than sixteen papers in International Journals and also presented sixteen papers in various National and international conferences. 